\title{
Acute kidney injury adjusted to volume status in critically ill patients: recognition of delayed diagnosis, restaging, and associated outcomes
}

This article was published in the following Dove Press journal: International Journal of Nephrology and Renovascular Disease 31 October 2016

Number of times this article has been viewed

\author{
Harout Yacoub' \\ Leen Khoury' \\ Youssef El Douaihy' \\ Chadi Salmane' \\ Jeanne Kamal' \\ Marc Saad ${ }^{2}$ \\ Patricia Nasr ${ }^{1}$ \\ Jared Radbel ${ }^{3}$ \\ Elie El-Charabaty' \\ Suzanne El-Sayegh' \\ 'Department of Medicine, Staten \\ Island University Hospital, Staten \\ Island, NY, USA; ${ }^{2}$ Division of Renal \\ Medicine, Emory University, Atlanta, \\ GA, USA; ${ }^{3}$ Department of Pulmonary \\ and Critical Care Medicine, Rutgers \\ Robert Wood Johnson Medical School, \\ New Brunswick, NJ, USA
}

Correspondence: Harout Yacoub Department of Medicine, Staten Island University Hospital, 475 Seaview Avenue, Staten Island, NY 10305, USA

Email hyacoub@norwell.edu
Abstract: Critically ill patients receive a significant amount of fluids leading to a positive fluid balance; this dilutes serum creatinine resulting in an overestimated glomerular filtration rate. The goal of our study is to identify undiagnosed or underestimated acute kidney injury (AKI) in the intensive care unit (ICU). It will also identify the morbidity and mortality associated with an underestimated AKI. We reviewed records of patients admitted to our institution (Staten Island University Hospital) between 2012 and 2013 for more than 2 days. Patients with end stage renal disease were excluded. AKI was defined using the Acute Kidney Injury Network criteria. The following formula was used to identify and restage patients with AKI: adjusted creatinine = serum creatinine $\times[$ (hospital admission weight $(\mathrm{kg}) 0.6+\Sigma$ (daily cumulative fluid balance $(\mathrm{L})) /$ hospital admission weight $\times 0.6]$. The primary outcome identified newly diagnosed AKI and those who were restaged. The secondary outcome identified associated morbidities. Seven-hundred and thirty-three out of 1,982 ICU records reviewed, were used. Two-hundred and fifty-seven (mean age $69.8 \pm 14.9$ ) had AKI, out of which $15.9 \%$ were restaged using the equation. Comparison of mean by Student's $t$-test showed no difference between patients who were restaged. Similarly, chi-square revealed no differences between both arms, except mean admission weight (lower in patients who were restaged), fluid balance on days 1, 2, and 3 (higher in the restaged arm), and the presence of congestive heart failure (more prevalent in the restaged arm). Of note, the mean cost of stay was US $\$ 150,562.82$ vs $\$ 197,174.63$ for same stage vs restaged, respectively, however, without statistical significance $(P=0.74)$. Applying the adjustment equation showed a modest (15.9\%) increase in the AKI staging slightly impacting outcomes (mortality, length, and cost of stay) without statistical significance.

Keywords: acute kidney injury, volume status, adjusted creatinine levels

\section{Introduction}

Small decrements in kidney function do not result in overt organ failure but are of significant clinical importance and impact on morbidity and mortality. Hence, the term acute kidney injury (AKI) has largely replaced acute renal failure.

One in five adults worldwide experience an AKI during hospitalization; these patients tend to have worse renal function at the time of discharge. In addition, mortality increases with the severity of an AKI. ${ }^{1}$

AKI is detected by an increase in serum creatinine (Scr). Since the volume of distribution of creatinine is equal to the total body water, ${ }^{2}$ a positive fluid balance during volume resuscitation will dilute Scr and thus, overestimate the glomerular filtration rate (GFR). ${ }^{3}$ This is of particular importance in the critical care setting. 
The goal of our study is to identify undiagnosed or underestimated AKI in the intensive care unit (ICU), regardless of diagnosis and identify the morbidity and mortality associated with it.

\section{Materials and methods Study design}

This is a single center retrospective study conducted at Staten Island University Hospital (SIUH). The study was approved by the North Shore-Long Island Jewish Health System Institutional Review board the approval number is IRB \#: SIUH13-657. The need for patient consent to participate was also waived by the IRB.

\section{Inclusion criteria}

We included adults (older than 18 years) who were admitted to SIUH ICU between 2012 and 2013 and stayed for at least 2 days in the ICU. End stage renal disease patients and patients with GFR less than 15 were excluded.

Baseline GFR was defined as the last calculated GFR prior to admission. GFR was calculated using the Modification of Diet in Renal Disease formula. Any readmission to the ICU for a single patient in the 2013 calendar year was not analyzed.

Patient data such as admission weight in $\mathrm{kg}$, Scr on ICU-days $0-3$, and cumulative fluid balance on ICU-days 1-3 were collected. Baseline Scr was defined as the first Scr measurement on ICU admission. One exception to this rule was that any patient admitted to the ICU for an elective surgery had the Scr on the day of the surgery as the baseline Scr. Day 1 Scr value was defined as next morning draw after admission/transfer to the ICU or the first day postoperatively if appropriate. Fluid balance was obtained from resident and intensivist documentation. If such data were missing or unreliable, the patient was excluded.

AKI staging was done using the Acute Kidney Injury Network (AKIN) Scr criteria. The AKIN urine output criteria were not used. Patients were defined as having precorrection AKI if they met the criteria at any point from days 1 to 3 , then staged accordingly. Patients were defined and staged as having post-correction $\mathrm{AKI}$ if they met AKIN definition after using the following formula:

Adjusted creatinine $=\mathrm{Scr} \times[$ (hospital admission weight $(\mathrm{kg}) \times 0.6+\Sigma$ (daily cumulative fluid balance $(\mathrm{L}))) /$

hospital admission weight $\times 0.6] .^{3}$

The primary outcome is to determine the number of patients classified as having AKI after correction for fluid status or those upgraded to a higher stage of AKI after correction to fluid status. Based on this data, patients were stratified into four groups: 1) no AKI precorrection/no AKI postcorrection; 2) no AKI precorrection/AKI postcorrection; 3) AKI precorrection/no increase in stage postcorrection; and 4) AKI pre-correction/increase in stage postcorrection.

Secondary outcomes are initiation of dialysis, in-hospital mortality, duration of ICU stay, duration of ventilation if intubated, admission length, cost of stay, and presence of a nephrology consultation within 72 hours of admission in groups $1-4$.

\section{Sample size}

Sample size was calculated based on available data that AKI can range from $20 \%$ to $50 \%$ in ICUs. A conservative estimate of $30 \%$ was used for this calculation. Based on previous studies, we expected to have approximately $25 \%$ of patients not diagnosed with AKI classified as such after applying the aforementioned fluid adjustment formula.

In order to yield an approximate final sample of 270 patients with AKI and a hypothesized number of 427 after using the adjustment formula, at least 900 charts of patients admitted to the ICU would be needed. This calculation would also allow a detection of a $20 \%$ difference in mortality between those not classified as AKI initially and then reclassified as AKI (31\%) compared to those who remained without AKI (12\%). The sample size has been calculated at a power of $80 \%$ and $P=0.05$ to detect differences in the AKI proportion before and after adjustment in creatinine as well as differences in mortality between these groups.

\section{Statistical analysis}

Mean and standard deviations were calculated on continuous variables and differences examined with Student's $t$-test or analysis of variance. Ordinal data for the subgroups were examined using Kruskal-Wallis for differences in medians. Categorical variables such as AKI class were examined for group differences on variables. Logistic regression was used to examine secondary outcomes adjusting for variables such as age, sex, ventilator status and/or vasopressor use during initial 3-day period, admission diagnosis, and presence of comorbidities such as hypertension, congestive heart failure (CHF), and diabetes (DM). Statistical significance was set at $P=0.05$, two-sided.

\section{Results}

A total of 1,982 ICU patients' charts were reviewed. Of them, 1,249 were excluded for incomplete data or failure to meet the inclusion criteria. Out of 733 patients, 257 (mean age $69.8 \pm 14.9)$ were found to have AKI. Of them, $15.9 \%(n=41)$ had higher AKI stages according to the adjustment equation. 
The baseline characteristics of our cohort were comparable with no statistical significance for mean age, incidence of hypertension and DM. However, CHF was more common in those who had higher AKI stages after applying the equation $\left(\chi^{2}=5.08, P=0.022\right)$. Although the sex distribution was marked by a heavier incidence of males in the arm with no AKI upstaging, this finding had no statistical significance. Mean weight was higher in the "same stage arm" with statistical significance (could be due to the effect of male sex predominance in that arm) (Table 1). GFR and creatinine were comparable at baseline and on the following 3 days.
Since the adjustment equation incorporates fluid status as a major factor, mean fluid balance was higher in the arm with upstage AKI on days 1, 2, and 3 (Table 2).

With regard to the interventions and characteristics of the hospital stay, there were no statistically significant differences. Nephrology services were equally consulted with $39.9 \%$ vs $36.6 \%$ in AKI same stage vs upstage, respectively $(P=0.5)$. The rate of hemodialysis was identical at $7.5 \%$ in both arms. Similarly, the use of vasopressors and the need for mechanical ventilation were comparable with no statistical significance between both arms (Table 3).

Table I Baseline characteristics

\begin{tabular}{|c|c|c|c|}
\hline Characteristic & $\begin{array}{l}\text { Same AKI stage proportion }(\%) \\
\text { or mean }( \pm S D)(n=2 \mid 6)\end{array}$ & $\begin{array}{l}\text { Higher AKI stage proportion }(\%) \\
\text { or mean }( \pm S D)(n=4 I)\end{array}$ & $\begin{array}{l}\text { Significance by }\left(\chi^{2}\right) \\
\text { or (Student's } t \text {-test) }\end{array}$ \\
\hline Sex & & & 0.074 \\
\hline Female & 84 (38.7\%) & 22 (53.7\%) & \\
\hline Male & $133(61.3 \%)$ & $19(46.3 \%)$ & \\
\hline Diabetes & $76(35.3 \%)$ & I 4 (35.0\%) & 0.966 \\
\hline Hypertension & 142 (65.7\%) & $28(70.0 \%)$ & 0.600 \\
\hline $\mathrm{CHF}$ & 51 (23.6\%) & $3(7.5 \%)$ & 0.022 \\
\hline Admission diagnosis & & & 0.595 \\
\hline Medical infectious & $64(29.5 \%)$ & $10(24.4 \%)$ & \\
\hline Medical noninfectious & 89 (41.0\%) & I4 (34.I\%) & \\
\hline Surgical infectious & 14 (6.5\%) & $3(7.3 \%)$ & \\
\hline Surgical noninfectious & $48(22.1 \%)$ & 13 (31.7\%) & \\
\hline Age & $70( \pm 15.3)$ & $71( \pm 12.6)$ & 0.577 \\
\hline Admission weight (kg) & $84.0( \pm 24.6)$ & $70.8( \pm 17.8)$ & 0.001 \\
\hline
\end{tabular}

Note: Data presented as mean, standard deviations, and proportions.

Abbreviations: $\mathrm{AKI}$, acute kidney injury; SD, standard deviation; $\mathrm{CHF}$, congestive heart failure.

Table 2 Kidney function and fluid balance characteristics

\begin{tabular}{llll}
\hline Characteristic & $\begin{array}{l}\text { Same AKI stage } \\
\text { mean }( \pm \mathbf{S D})(\mathbf{n = 2 1 6})\end{array}$ & $\begin{array}{l}\text { Higher AKI stage } \\
\text { mean }( \pm \mathbf{S D})(\mathbf{n = 4})\end{array}$ & $\begin{array}{l}\text { Significance } \\
\text { Student's t-test }\end{array}$ \\
\hline Baseline GFR & $60.6( \pm 32.3)$ & $60.7( \pm 38.3)$ & 0.985 \\
Scr at baseline (day 0) & $2.0( \pm I .9)$ & $1.5( \pm 0.9)$ & 0.152 \\
Scr day I & $1.9( \pm I .4)$ & $1.7( \pm 1.1)$ & 0.360 \\
Scr day 2 & $1.7( \pm I .2)$ & $1.8( \pm 1.2)$ & 0.729 \\
Scr day 3 & $1.6( \pm I .1)$ & $1.9( \pm 1.2)$ & 0.072 \\
Fluid balance day I (L) & $2.1( \pm 2.6)$ & $3.4( \pm 3.3)$ & 0.005 \\
Fluid balance day 2 & $1.3( \pm 2.0)$ & $2.6( \pm 2.5)$ & 0.000 \\
Fluid balance day 3 & $0.5( \pm I .8)$ & $1.6( \pm 2.3)$ & 0.002 \\
\hline
\end{tabular}

Note: Data presented as mean, standard deviations, and proportions.

Abbreviations: AKI, acute kidney injury; Scr, serum creatinine; GFR, glomerular filtration rate; SD, standard deviation.

Table 3 Inpatient interventions

\begin{tabular}{lll}
\hline & Same AKI stage & Higher AKI stage \\
\hline Nephrology consult on days I-3 & $85(39.9 \%)$ & $\chi^{2}(\boldsymbol{P}$-value $)$ \\
Mechanical ventilation & $120(56.1 \%)$ & $15(36.6 \%)$ \\
Vasopressors & $58(26.9 \%)$ & $25(61.0 \%)$ \\
Initiation of HD & $16(7.5 \%)$ & $13(31.7 \%)$ \\
\hline
\end{tabular}

Note: Data presented as number of occurrences and proportions. Abbreviations: AKI, acute kidney injury; HD, hemodialysis. 
Table 4 Mortality and secondary outcomes

\begin{tabular}{|c|c|c|c|}
\hline & Same AKI stage (SD) & Higher AKI stage (SD) & $\begin{array}{l}\text { Student's t-test } P \text {-value } \\
\text { or } \chi^{2}(P \text {-value })\end{array}$ \\
\hline ICU stay in days & $8(8)$ & $8(6)$ & 0.98 \\
\hline Admission length & $17(13)$ & $19(17)$ & 0.45 \\
\hline Cost of stay & US\$14I,535.95 (\$132, |25.75) & US\$188,630.73 (\$183 207.83) & 0.07 \\
\hline $\begin{array}{l}\text { Days on ventilation (only for ventilation-dependent) } \\
\text { ( } n=120 \text { vs } 25)\end{array}$ & $6(10)$ & $6(6)$ & 0.90 \\
\hline Mortality & $46(21.6 \%)$ & II (26.8\%) & $0.54(.46)$ \\
\hline
\end{tabular}

Note: Data presented as number of occurrences, standard deviations, and proportions.

Abbreviations: AKI, acute kidney injury; SD, standard deviation; ICU, intensive care unit.

Length of stay and ICU days were comparable. The mean length of hospital stay was 17 vs 19 days for same stage vs upstage groups, respectively, with no statistical significance by Student's $t$-test $(P=0.45)$. Similarly, the ICU stay was approximately 8 days in both groups $(P=0.98)$. The mean number of days on mechanical ventilation in those who needed ventilation was identical as well (6 days $P=0.90$ ). Despite a difference exceeding US $\$ 40,000$ in the cost of hospital stay between the two groups (higher in the upstaged group), this difference was not statistically significant $(P=0.07)$. Lastly, mortality was slightly higher in the upstaged group (26.8\%) compared to same stage (21.6\%) with no statistical significance $(P=0.46)$ (Table 4$)$.

\section{Discussion}

AKI in critical care setting gained a lot of attention over the past decade mostly due to increased mortality with slight increase in creatinine levels. ${ }^{4,5}$ Conservative estimates report $25 \%-30 \%$ of ICU patients develop AKI with mortality rate exceeding $50 \%$ in those who require renal replacement therapy. ${ }^{6,7}$ Sepsis, pulmonary, and cardiac dysfunction further complicate the management of AKI. Despite that these conditions have an equally important role in determining the prognosis of a critical patient, their management differs significantly and further complicates fluid management in these patients.

There is growing evidence that fluid accumulation is independently associated with worse ICU outcomes and increased mortality in patients with kidney injury. ${ }^{8-10}$ The main suggested mechanism is increased intra-abdominal pressure and compression of the inferior vena cava causing renal congestion and compromised venous return. ${ }^{11,12}$ Fluid accumulation also dilutes Scr and delays AKI diagnosis. Macedo et al indicated that $50 \%$ of the time AKI diagnosis was missed or delayed in the ICU because of fluid accumulation and suggested the formula used in our study to improve early recognition and severity assessment. ${ }^{3}$
The observed median cumulative fluid balance increased to reach $6.5 \mathrm{~L}$, and the difference between adjusted and crude Scr was significantly higher at each time point and progressively increased to reach $0.65 \mathrm{mg} / \mathrm{dL}$. Eventually $25 \%$ patients met criteria for a late recognition to estimate severity progression of AKI.

In another large cohort study, Thongprayoon et al found that Scr adjustment for fluid balance could result in a more accurate detection of AKI cases. ${ }^{13}$ They also indicated that the risk of 60-day mortality in patients who met AKI criteria (after adjustment) was significantly higher than in patients without AKI (odds ratio 2.00; 95\% confidence interval 1.25-3.11). The same was true in cardiac surgical patients where Moore et al concluded that patients with AKI diagnosed only after correction for the effect of a positive fluid balance on Scr concentration have worse renal, ICU, and hospital outcomes. ${ }^{14}$

It is confirmed that adjusting to volume status identifies more AKI cases in an earlier time frame. ${ }^{3}$ The effect of upstaging previously diagnosed AKI is still subject to debate. In our study, we reported a cohort study at a community hospital critical care unit. Our main goal is to identify the relevance of adjusting Scr levels to fluid balance and its effect on overall outcome for these patients. The main focus is comparing the patients who were identified as having AKI without change of the AKI stage to those who were newly diagnosed or were upstaged AKI. This differs from other previous reports describing the effect of fluid balance on newly diagnosed cases only. ${ }^{13,14}$

Our study was limited by several factors. This is a retrospective observational study that mainly relied on intensivist and resident documentation of acuity, diagnosis, and comorbid condition. Although we identified acute respiratory failure cases requiring mechanical ventilation, we did not identify acute respiratory distress syndrome (ARDS) cases specifically. ARDS management requires conservative fluid management and permissive hypercapnia protocol which effects acutely injured kidney. ${ }^{15,16}$ This study was not designed to evaluate 
the time difference in diagnosing AKI after adjustment for fluid balance. It is reported that such adjustment may lead to earlier detection, but the effect of early detection of AKI is yet to be proven by prospective studies. Another limitation of our study is the lack of intra-abdominal pressure measurement. Fluid overload-induced intra-abdominal hypertension plays a significant role in the pathophysiology of renal injury predisposing to a prerenal state ${ }^{11,12}$ and further worsening the preexisting injury. Measurement of intra-abdominal pressure usually requires urinary catheter insertion, which is not the standard of care for all ICU admissions.

Our study has many strengths. The study sample is a good representation of medical and surgical ICUs taking into consideration the admitting diagnosis and presence of infectious etiology. Our sample was comparable in baseline characteristics, including age and hypertension. There was no difference in admission diagnosis (medical, surgical, infectious or noninfectious) between the two groups $(P=0.595)$. The fact that we had more heart failure cases in the same stage group ( $23.6 \%$ vs $7.5 \% P=0.22)$ could be explained by a more conservative fluid management approach in this subgroup. The mean fluid balance was expectedly higher and the mean Scr was lower in the upstaged group (Table 2). The accurate documentation of fluid balance by the intensivist and correlation with the resident notes made our fluid accumulation calculations reliable and accurate.

\section{Conclusion}

In our sample, applying the adjustment equation showed a modest $15.9 \%$ increase in the AKI staging slightly impacting outcomes (mortality, length, and cost of stay) without statistical significance. However, adjusting for volume status remains essential for earlier and more accurate diagnosis of renal injury in the critical care setting. Prospective studies are needed to further assess the effect of Scr adjustment to volume status on critical care management and overall outcome.

\section{Acknowledgments}

1. Paul Catella, MD, Department of Medicine, Staten Island University Hospital, Staten Island, NY, USA - data collection;

2. Ahmed Mahgoub, MD, Department of Medicine, Staten Island University Hospital, Staten Island, NY, USA - data collection;
3. Jawalant Modi, MD, Department of Nephrology Indiana University-Purdue University, Indianapolis, IN, USA data collection.

\section{Disclosure}

The authors have no conflicts of interest to declare and the study received no funding.

\section{References}

1. Hoste EA, Bagshaw SM, Bellomo R, et al. Epidemiology of acute kidney injury in critically ill patients: the multinational AKI-EPI study. Intensive Care Med. 2015;41(8):1411-1423.

2. Bjornsson TD. Use of serum creatinine concentrations to determine renal function. Clin Pharmacokinet. 1979;4(3):200-222.

3. Macedo E, Bouchard J, Soroko SH, et al. Fluid accumulation, recognition and staging of acute kidney injury in critically-ill patients. Crit Care. 2010;14(3):R82.

4. Chertow GM, Burdick E, Honour M, Bonventre JV, Bates DW. Acute kidney injury, mortality, length of stay, and costs in hospitalized patients. J Am Soc Nephrol. 2005;16(11):3365-3370.

5. Vaara ST, Pettila V, Kaukonen KM, et al. The attributable mortality of acute kidney injury: a sequentially matched analysis*. Crit Care Med. 2014;42(4):878-885.

6. Uchino S, Kellum JA, Bellomo R, et al. Acute renal failure in critically ill patients: a multinational, multicenter study. JAMA. 2005;294(7):813-818.

7. Johansen KL, Smith MW, Unruh ML, et al. Predictors of health utility among 60-day survivors of acute kidney injury in the Veterans Affairs/ National Institutes of Health Acute Renal Failure Trial Network Study. Clin J Am Soc Nephrol. 2010;5(8):1366-1372.

8. Bouchard J, Soroko SB, Chertow GM, et al. Fluid accumulation, survival and recovery of kidney function in critically ill patients with acute kidney injury. Kidney Int. 2009;76(4):422-427.

9. Mehta RL, Bouchard J, Soroko SB, et al. Sepsis as a cause and consequence of acute kidney injury: Program to Improve Care in Acute Renal Disease. Intensive Care Med. 2011;37(2):241-248.

10. Heung M, Wolfgram DF, Kommareddi M, Hu Y, Song PX, Ojo AO. Fluid overload at initiation of renal replacement therapy is associated with lack of renal recovery in patients with acute kidney injury. Nephrol Dial Transplant. 2012;27(3):956-961.

11. Doty JM, Saggi BH, Blocher CR, et al. Effects of increased renal parenchymal pressure on renal function. J Trauma. 2000;48(5):874-877.

12. Doty JM, Saggi BH, Sugerman HJ, et al. Effect of increased renal venous pressure on renal function. J Trauma. 1999;47(6):1000-1003.

13. Thongprayoon C, Cheungpasitporn W, Srivali N, Ungprasert P, Kittanamongkolchai W, Kashani K. The impact of fluid balance on diagnosis, staging and prediction of mortality in critically ill patients with acute kidney injury. $J$ Nephrol. 2016;29(2):221-227.

14. Moore E, Tobin A, Reid D, Santamaria J, Paul E, Bellomo R. The Impact of Fluid Balance on the Detection, Classification and Outcome of Acute Kidney Injury After Cardiac Surgery. J Cardiothorac Vasc Anesth. 2015;29(5):1229-1235.

15. Kallet RH, Jasmer RM, Luce JM, Lin LH, Marks JD. The treatment of acidosis in acute lung injury with tris-hydroxymethyl aminomethane (THAM). Am J Respir Crit Care Med. 2000;161(4 Pt 1):1149-1153.

16. Kramer AA, Postler G, Salhab KF, Mendez C, Carey LC, Rabb H. Renal ischemia/reperfusion leads to macrophage-mediated increase in pulmonary vascular permeability. Kidney Int. 1999;55(6):2362-2367. 
The International Journal of Nephrology and Renovascular Disease is an international, peer-reviewed open access journal focusing on the pathophysiology of the kidney and vascular supply. Epidemiology, screening, diagnosis, and treatment interventions are covered as well as basic science, biochemical and immunological studies. The manuscript management system is completely online and includes a very quick and fair peer-review system, which is all easy to use. Visit http://www. dovepress.com/testimonials.php to read real quotes from published authors.

Submit your manuscript here: https://www.dovepress.com/international-journal-of-nephrology-and-renovascular-disease-journal 